\title{
EDITORIAL
}

Klin. Pädiat. 198 (1986) 431-434

(C) F. Enke Verlag Stuttgart

\section{Regionalisierung der perinatalen Medizin Wohin gehört die Neonatologie?*}

\author{
V.v. Loewenich
}

Abteilung für Neonatologie, Zentrum der Kinderheilkunde der Johann Wolfgang Goethe-Universität, Frankfurt/Main (Leiter: Prof. Dr. V. v. Loewenich)

1967 gründete Erich Saling die weltweit erste Gesellschaft furr Perinatale Medizin. Er vereinigte in dieser Gesellschaft diejenigen Disziplinen, die perinatal, $\mathrm{d}$ h. um die Geburt herum, fuir das Wohl des Kindes zu sorgen haben, nämlich die Frauenheilkunde mit ihrer schon alten Subspezialität Geburtshilfe auf der einen und die Kinderheilkunde mit ihrer damals eben erst im Entstehen begriffenen Subspezialität Neonatologie auf der anderen Seite. Dieses Beispiel machte in allen Kulturstaaten Schule. Heute ist die perinatale Medizin ein international eingefuihrtes Fachgebiet. Dennoch unterscheidet sie sich in einer Hinsicht von allen anderen Fächern: Sie ist ein kooperatives Fach, das auf zwei Säulen ruht, nämlich auf den oben erwähnten Fächern Geburtshilfe und Neonatologie. Es hat deshalb auch bis heute kein einziges ,Exemplar eines Perinatologen” gegeben, der das ganze Spektrum dieser Dizsiplín ausübt und beherrscht. Es gibt nur den perinatalmedizinisch ausgerichteten Geburtsmediziner und den perinatalmedizinisch ausgerich teten Kinderarzt, nämlich den Neonatologen. Die Kooperation beider ist die Chance der perinatalen Medizin.

Praktische Ansätze zur perinatalmedizinischen Zusammenarbeit hatte es indessen schon früher gegeben. So wurde an der Basler Universitäts-Frauenklinik bereits in der ersten Hälfte der 60er Jahre eine Neugeborenen-Intensivstation eingerichtet, allerdings unter der Regie der Frauenklinik, wenngleich interdisziplinär besetzt mit einem Frauenarzt, einem Kinderarzt und einem Anästhesisten. 1965 kam mein pädiatrischer Lehrer, Otto Hövels, mit einem sehr pragmatischen Ansatz nach Frankfurt/M.: Bereits während seiner Zeit als Direktor der Städtischen Kinderklinik in Nümberg hatte er epidemiologische Studien zur Säuglingssterblichkeit angestellt. Dabei fand er, daß sich die meisten Säuglingstodesfälle am ersten Lebenstag ereigneten und hier wieder innerhalb der ersten Lebensstunden. Die von ihm hieraus gezogene Konsequenz war überaus einfach und überaus einleuch tend: Wenn man die Säuglingssterblichkeit entscheidend senken will, dann muß man sich schwergewichtig um das Neugeborene direkt nach der Geburt kümmern. Auch heute liegt der Schwerpunkt der Säuglingssterblichkeit noch im Neugeborenenal ter. So ereigneten sich 1984 in Hessen $43 \%$ aller Säuglingssterbe fälle innerhalb der ersten Lebens. woche (14). Der Vorschlag von Otto Hövels anläßlich seiner Berufung nach Frankfurt/M. lautete folgerichtig, eine von Kinderheilkunde und Geburtshilfe gemeinsam zu be-

* Herrn Prof. Dr. med. Otto Hövels zum 65. Geburtstag gewidmet treibende Neugeborenen-Intensiveinheit im gerade entstehenden Neubau der Frauenklinik einzurich ten. Otto Hövels lief mit diesem Vorschlag bei seinem geburtshilflichen Kollegen, Otto Kaeser, offene Türen ein. Der Vorschlag wurde in die Tat umgesetzt (16). Seit Anfang 1972 arbeiten Neonatologie mit einer Neugeborenen-Intensivstation und Geburtshilfe innerhalb eines Hauses, lediglich durch eine Türe getrennt, störungsfrei und erfolgreich zusammen. Die Neugeborenen-Intensivstation ist allerdings nicht, wie ursprünglich geplant, eine interdisziplinär betriebene Einheit. Diese Idee erwies sich im Laufe der Jahre als nicht realisierbar. Geburtshelfer konnten in die Arbeit dieser Station nur als Hospitanten einbezogen werden. Die Aufgaben der Geburtsmedizin auf der einen Seite und der Neonatologie auf der anderen Seite sind zu vielfältig, als daß ein gemeinsames Arbeiten hier realisierbar gewesen wäre. Was hingegen heranwuchs, war eine engverzahnte Kooperation, die mit der gegenseitigen Konsultation bei pränatal erkannten Problemen beginnt und mit der Entlassung des Kindes endet.

\section{Gründe für die Einrich tung perinatalmedizinischer Zentren:}

Die gegenwärtige Situation der perinatalen Medizin in der Bundesrepublik Deutschland ist die folgende : Die Mehrzahl aller Kinder wird in einer sehr großen Zahl meist kleiner geburtshilflicher Abteilungen geboren. Dies gilt in der Regel auch für Kinder mit einem sehr hohen Mortalitä ts- und Morbiditätsrisiko, hier insbesondere die sehr kleinen Frühgeborenen. Die sehr schlechte Prognose solcher Kinder hat dazu gefuihrt, daß mittlerweile fast flächendeckend Transportsysteme eingerich tet wurden, bei denen ein kinderklinisches Team Risikokinder abholt und in eine Kinderklinik transportiert. Vor der Einrich tung solcher Transportsysteme waren die Unterschiede in der Prognose transportierter gegenüber in einem Zentrum geborener Kinder sehr kraß (9). Aber auch heute lassen die Ergebnisse noch zu wünschen übrig $(18,23)$. Eine gewisse Verbesserung läßt sich dadurch erreichen, daß man sich nicht auf einen Rettungstransport un ter In tensivbehandlungsbedingungen beschränkt, sondern daß kinderärztliche Teams bereits zur Geburt von Risikokindern anwesend sind, um sofort tätig zu werden $(18,21)$. Der Aufwand solcher vorverlegter stationärer Behandlung ist indessen gewaltig und muß als ausgesprochen unökonomisch betrach tet werden. Das mitunter stundenlange Warten eines kinderklinischen Teams fern vom eigentlichen Wirkungsort dünnt die Versorgung der eigenen stationären $\mathrm{Pa}$ - 
tienten aus. Kommt es gleichzeitig an verschiedenen Orten zu Risikogeburten, so ist das ,zu spät gekommene" Kind möglicherweise in seinen Uberlebenschancen erheblich beein trächtigt. Dies hat dazu geführt, daß man sowohl von ärztlicher $(2,6,9,12,13,17,18,19,22,23,24,25,26$, $27)$ als auch von politischer $(11,14)$ Seite die Einrichtung perinatalmedizinischer Zentren fordert. Diese sollen nach dem Vorbild anderer Länder (z.B. Finnland, siehe aber auch Abb. 1) für Geburten mit besonders hohem Risiko zur Verfügung stehen. Hierzu zählen Frühgeburten vor der 33. Schwangerschaftswoche, Mehrlings-Frühgeburten, Geburten von Kindern mit bereits pränatal diagnostizierbaren Erkrankungen oder Gefahrenzuständen. Gerade das Beispiel Finnland zeigt, daß eine rechtzeitige Selektion solcher Geburten, die etwa $3 \%$ aller Geburten ausmachen, durchaus möglich ist. Innerhalb der deutschen Pädiatrie wurde in letzter Zeit diskutiert, ob pernatalmedizinische Zentren in der Tat effizienter seien als die bisher in der Bundesrepublik Deutschland übliche Trennung zwischen Geburtsmedizin und Kinderheilkunde. Es liegen mittlerweile soviele Studien darüber vor, daß die Zentralisation von Risikogeburten in perinatalmedizinische Zentren sowohl die Mortalität als auch die Morbidität in einem so nennenswerten Maße zu senken im. stande ist, daß über diese Frage nicht mehr diskutiert werden muß $(1,3,4,7,8,9,10,12,13,15,17,19,20,25$, $26,27)$.

Tab. 1 Einfluß der Regionalisierung extremer Frühgeburten $\{\mathrm{Ge}$ burtsgewicht unter $1500 \mathrm{~g}$ ) (Hessische Perinatalstudie 1983, 1984 und 1985 (1. Hälfte)) $; n=488$. Zahlen aus (15)

\begin{tabular}{lr}
\hline Perinatale Sterblich keit & \\
\hline Zentrum JWGUFM & 18 von $140(13 \%)$ \\
übriges Hessen & 111 von $348(32 \%)$ \\
\hline
\end{tabular}

$p<0,0001$

Tab. 1 zeigt anhand der Hessischen Perinatalstudie, daß die Sterblichkeit sehr kleiner Frühgeborener, $d \boldsymbol{h}$. von Kindern mit einem Geburtsgewicht unter $1500 \mathrm{~g}$, in einem Zentrum um den Faktor 2,5 niedriger liegt als im Durchschnitt des übrigen Bundeslandes. Da man schwerlich unterstellen kann, in unserem Zentrum würde um den Faktor 2,5 bessere Arbeit geleistet, bleibt nur die eine Interpretation, daß das signifikant bessere Ergebnis ausschließlich der Organisationsform ,perinatalmedizinisches Zentrum" zuzusch reiben ist.

Was für die Mortalität gilt, gilt ebenso für die Morbidität. Diese ist am einfachsten und objektivsten an der Häufigkeit in traventrikulärer Blutungen zu messen. Auch hier haben wir eine bessere Prognose derjenigen Kinder nachweisen können, die innerhalb eines Zentrums geboren worden waren und die nicht transportiert werden mußten $(3,17)$. Es ist dabei offen, ob der Transport selbst, z.B. au fgrund mechanischer Insulte (5) schädigend wirkt, oder ob es die "Kondition Transport" oder die „Kondition ou tborn" ist. Da unsere eigenen „Outborn"-Zahlen dank der sehr gut funktionierenden Regionalisierung von Hochrisikogeburten im Raum Frankfurt relativ klein sind, haben wir vergleichbare Zahlen
Tab. 2 (Zahlen aus 3, 7, 10)

\begin{tabular}{lll}
\hline $\begin{array}{l}\text { S. Francisco } 1981 \\
\text { Houston } 1982 \\
\text { Frankfurt 1984 }\end{array}$ & & \\
& & \\
Outborn & $50 \% \pm 6 \%$ \\
Inborn & $18 \% \pm 6 \%$ & $(90 \%-C L)$
\end{tabular}

$p<10^{-6} \quad(n=319)$

IVH $=$ intraventrikuläre Blutung

VLBWI = sehr kleine Früh geborene $11500 \mathrm{~g}$ Geburtsgewicht und weniger)

Tab. 3 (Zahlen aus 3, 7, 10)

Reduktion der Hạ̈ufigkeit intraventrikulärer Blutungen bei ,"kleinen " Frühgeborenen durch

Regionalisierung der Geburt

um den Faktor $2,8(p=0,025)$

oder 1,8 bis 4,7 fach $(95 \%$ - VB\})

S. Francisco 1981, Houston 1982, Frankfurt a.M. $1984(n=319)$

verschiedene Zentren zusammen durchgerechnet $(3,7,10)$ Das Ergebnis zeigen die Tabellen 2 und 3.

\section{Wohin gehört die Neonatologie?}

Wir können zunächst einmal festhal ten, daß eine nach heu tigem Wissen optimale Versorgung von Hochrisikogeburten nur dort möglich ist, wo neonatologische In tensivmedizin und Geburtsmedizin unmittelbar einander zugeordnet arbeiten. Dies wurde oben hinreichend belegt. Man wird diesen Tatsachen von seiten der Pädiatrie Rechnung zu tragen haben.

Auf der anderen Seite ist die Neonatologie ein sehr wesentlicher Bestandteil der Kinderheilkunde. Die Belegungszahlenब in den einzelnen Abteilungen unserer Kinderkliniken sprechen hier eine deutliche Sprache. Es muß nicht näher begründet werden, daß das besonders kleine Kind nicht weniger, sondern mehr in die Hand des Kinderarztes gehört als jedes andere Kind. Es hat auch bisher nie jemand emsthaft infrage gestellt, daß die Behandlung Neugeborener und Früh geborener Sache der Pädiatrie ist.

Schwierigkeiten ergeben sich indessen mitun ter bei der prak tischen Realisierung. Nur allzu häufig sind Frauenklinik und Kinderklinik räumlich weit voneinander getrennt. Dies hat an manchen Orten dazu geführt, daß Frauenkliniker eine neonatologische Abteilung an der Frauenklinik fordern. Die Begründung dieser Forderung ist nach den obigen ${ }^{\circ}$ Darlegungen nicht schwierig. Dennoch wird von frauenklinischer Seite dabei häufig übersehen, daß die Neonatologie so etwas ist wie die Allgemeinmedizin eines bestimmten Lebensalters. Zwar hat die Neonatologie ihr großes Schwergewicht bei der Intensivbehandlung. Dies ist aber keineswegs das ganze Spektrum der Neonatologie, was aus der Sicht anderer Fächer mitunter vergessen wird. Die kunstgerechte Behandlung eines Neugeborenen schließt die Mitwirkung so gut wie aller weiteren pädiatrischen Subspeziali- 


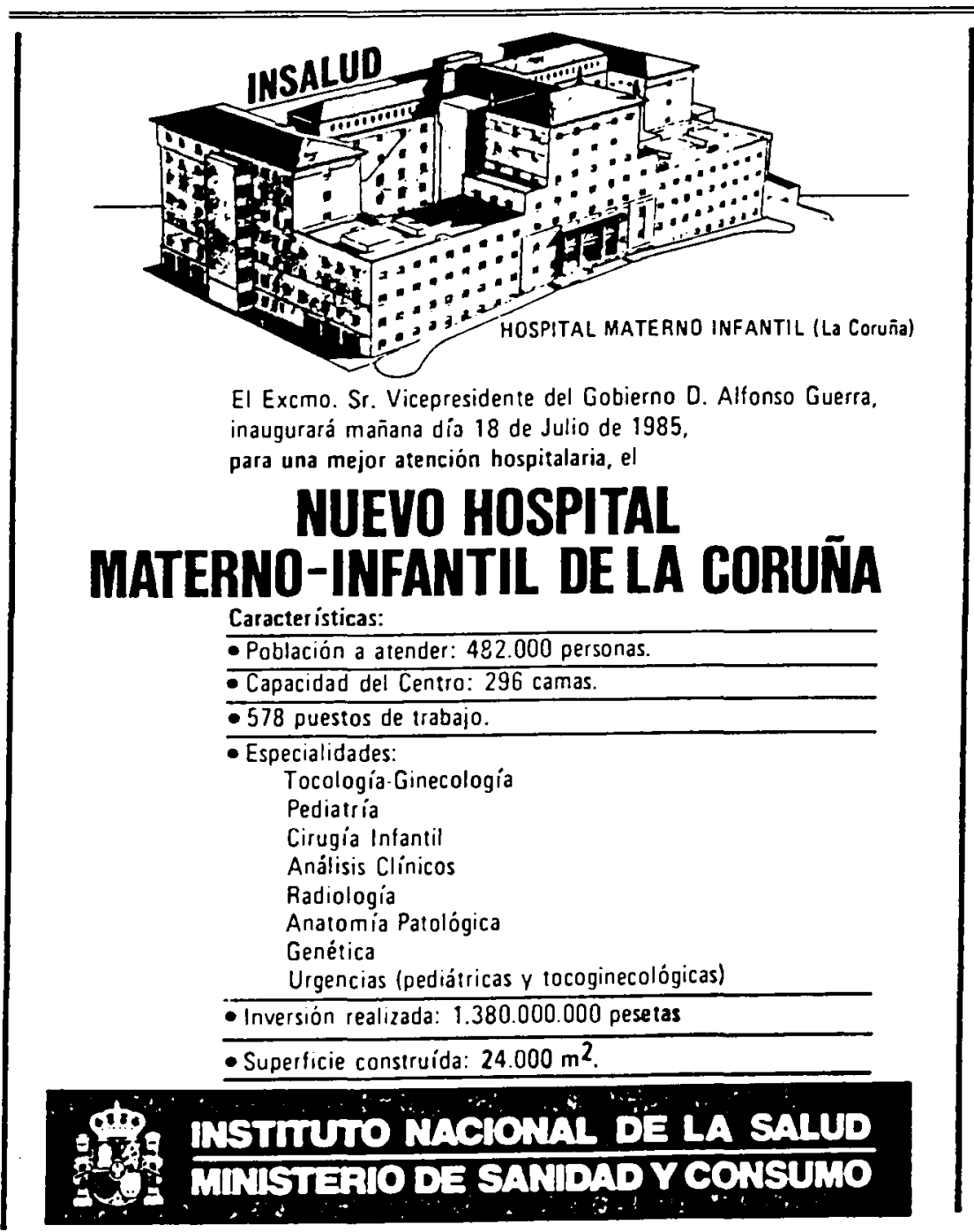

Abb. 1 Mutter-Kind-Krankenhaus in La Coruña.

Übersetzung des Testes unterhalb der großen Überschrift:

Charak teristika:

zu versorgende Einwohnerzahl: 482.000

Kapazität des Zentrums: 296 Betten

587 Arbeitsplätze

Spezialitäten:

Geburtshilfe - Gynäkologie; Kinderheil-

kunde; Kinderchirurgie; Laboratorien;

Radiologie; path. Anatomie; Humangenetik:

Notfallbehandlung (kinder- und fraven-

klinisch)

getätigte Investitionen: 1.380 .000 .000 Peseten

= ca. DM 96 Mio.

Raumangebot: $24.000 \mathrm{~m}^{2}$

(aus El Pais vom 17. Juli 1985) täten ein. Sie schließt auch und ganz besonders die Mitwirkung der Kinderchirurgie ein. Die weite räumliche Trennung von Neonatologie und übriger Pädiatrie bringt deshalb erhebliche organisatorische Probleme, u.U. sogar einen Qualitätsverlust mit sich. Kinderradiologische, kinderkardiologische, kinderneurologische Untersuchungen müssen rasch und vollständig durchführbar sein. Es wäre widersinnig, wenn man zu diesen Zwecken hochgefährdete Neu- und Frühgeborene, die nur zur Vermeidung eines postnatalen Transportes in einem Zentrum geboren wurden, dann zwecks weiterer diagnostischer und therapeutischer Maßnahmen transportieren würde.

Ein weiterer Gesichtspunkt ist nicht gering zu achten: Der Neonatologe benötigt nicht nur den steten Gedankenaustausch mit dem Geburtsmediziner, den ihm die Organisationsform eines perinatalmedizinischen Zentrums bietet und der sich, wo realisiert, als sehr fruchtbar erwiesen hat. Der Neonatologe ist genauso auf den praktisch-klinischen und wissenschaftlichen Gedankenaustausch mit den Vertretern der übrigen pädiatrischen Spezialitäten angewiesen.
Konsequenzen:

Die Neonatologie benötigt einerseits den Hintergrund der kompletten Pädiatrie und muß andererseits in nächstmöglicher Nachbarschaft zur Geburtshilfe arbeiten, um so effektiv zu sein, wie dies heute möglich ist. Daraus ergibt sich der logische Schluß, daß mindestens an Schwerpunkten Kinderklinik und Frauenklinik aneinandergebaut werden müssen. Die Stoßstelle beider Institutionen sind Kreißsaal und Neugeborenen-Intensivstation. Da große Kinderkliniken aber auch Intensivtherapie bei Kindern jenseits des Neugeborenenalters treiben müssen, ist es nichts weniger als naheliegend, die übrige pädiatrische Intensivmedizin gleichfalls in Nachbarschaft zur Neugeborenen-Intensivmedizin unterzubringen. Auf den humanitären und psychohygienischen Aspekt der Unterbringung von Mutter und Kind unter dem gleichen Dach sei nur am Rande verwiesen.

Selbstverständlich wird es nicht überall möglich sein, diese Erkenntnisse sogleich architektonisch in die Tat umzusetzen. Andererseits hat seit einigen Jahren ein Umdenken 
in der Krankenhausplanung eingesetzt. Die Euphorie der $60 \mathrm{er} \mathrm{Jahre}$, in denen viel $\mathrm{zu}$ viele Krankenhausbetten an viel $\mathrm{zu}$ vielen Orten eingerichtet worden waren, ist verflogen. Länder und Kommunen beschäftigen sich mit einer „Flurbereinigung” des Krankenhauswesens. Aufgabe der deutschen Pädiatrie ist es heute, bei allen Reorganisationen des Krankenhauswesens deutlich $\mathrm{zu}$ fordern, daß mindestens an Schwerpunkten der Patientenversorgung leistungsfähige Frauenkliniken und Kinderkliniken einander engstmöglich zugeordnet werden. Daß dies auch in wirtschaftlich weit weniger begünstigten Ländern möglich ist, mag die Abb. 1 demonstrieren. Die Bundesrepublik Deutschland hebt sich hier im Vergleich mit Nachbarländern negativ ab. Hierzulande ist es sogar zur Demontage von Einrichtungen gekommen, die sich mit wenig Mitteln zum perinatalmedizinischen Zentrum hätten ausbauen lassen $(21,22)$. Wo es nicht anders möglich ist, sollte mindestens innerhalb des gleichen Klinikums die Neugeborenen-Intensivstation den Entbindungsräumen engstmöglich zugeordnet werden. Es muß aber betont werden, daß es nicht das Gebot der Stunde ist, nach möglichst billigen Kompromissen zu suchen. Die Pädiatrie hat mit guten Gründen seit jeher die Prävention auf ihre Fahnen geschrieben. Es ist daher unsere Aufgabe als Kinderärzte, rechtzeitig und deutlich die richtigen Zukunftsperspektiven aufzuzeigen.

\section{Literatur}

1 Anderson, C., S. Aladjem, O. Ayuste, C. Caldwell, M. Ismail: An analysis of maternal transport within a suburban metropolitan region. Am. J. Obstet. Gynecol. 140 (1981) 499-504

2 Beck, L., E. Maier, E. Schmidt, J.J. Rohde, Statistisches Bundesamt: Mütter- und Säuglingssterblichkeit. Bd. 67 der Schriftenreihe des Bundesministers für Jugend, Familie und Gesundheit. Stuttgart/Berlin/Köln/Mainz 1978 (W. Kohlhammer)

3 Bielicki, M., V. von Loewenich, E. Halberstadt, H. Grau: Intraventrikuläre Blutungen bei sehr kleinen Frühgeborenen. In: $\mathbf{S}$. Kowalewski (Hrsg.): Pädiatrische Intensivmedizin VI, INA Bd. 47 Thieme, Stuttgart-New York 1984, S. 150-152

4 Boehm, F.H., M.F. Haire: Onc-way maternal transport: A evolving concept. Inpatient services. Am. J. Obstet. Gynecol. 134 (1979) $484-489$

5 Boenisch, H., W. Gaden, D. Mau, U. Gurbandt, H. O. Teuteberg, H. Braun, H.J. Beermann: Mechanische Belastung Neugeborener bei Inkubatortransporten. Mschr. Kinderheilk. 133 (1985) 471-475

6 Burghard, H., U. Töllner: Transport des Risikoneugeborenen oder der Risikoschwangeren? Dtsch. Med. Wschr. 106 (1981) 10191021

7 Clark, C.E., R.I. Clymann, R.S. Roth, S.H. Sniderman, B. Lane, R.A. Ballard: Risk factor analysis of intraventricular hemorrhage in low birthweight infants. J. Pediatr. 99 (1981) 625628

8 Cordero, L., C. Backes, F. Zuspan Very low birth weight infant: I. Influence of place of birth on survival. Am. J. Obstet. Gynecol. 143 (1982) 533-537

9 Eyring, $R ., V$. von Loewenich: Statistisch-epidemiologische Untersuchungen über die negativen Auswirkungen des Fehlens eines Neugeborenen-Transportsystems. In: P. Lemburg (Hrsg.):
Pädiatrische Intensivmedizin II, INA Bd. 27. Thieme, StuttgartNew York 1981, S. 195-199

10 Garcia-Prats, J.A., R.S. Procianoy, J.M. Adams, A.J. Rudolp: The hyaline membrane disease - intraventricular hemorrhage relationship in the very low birth weight infant. Perinatal aspects. Acta Paediatr. Scand. 71 (1982) 79-84

11 54. "GMK" (54. Konferenz der für das Gtsundheitswesen zuständigen Minister und Senatoren der Länder) 8./9. Oktober 1985 in Stuttgart

12 Glies, H.R.: Maternal transport. Clinics in Obstetrics and Gynaecology 6 (1979) 203-214

13 Harris, T.R., J. Isaman, H.R. Giles: Improved neonatal survival through maternal transport. Obstet. Gynecol. 52 (1978) $294-$ 300

14 Hessische Landesregierung, Drucksache 11/53.33 vom 17.2.86 (Wiesbaden)

15 Hessische Perinatalstudie 1983, 1984, 1985

16 Loewenich, V.von, D. Berg: Planung und Organisation einer interdisziplinären Intensivbehandlungseinheit für Neugeborene. In H.W. Opderbecke (Hrsg.): Planung Organisation und Einrich tung von Intensivbehandlungseinheiten am Krankenhaus. Anaesthesiol. Wiederbelebg; Bd. 33. Springer, Berlin, Heidelberg, New York 1969 S. 116-120

17 Loewenich, V. von, M. Bielicki E. Halberstadt, H. Grau: Wie weit ist die intraventrikuläre Blutung sehr unreifer Frühgeborener vermeidbar? In: J.W. Dudenhausen, E. Saling (Hrsg.): Perinatale Medizin X, Thieme, Stuttgart-New York 1984 , S. 308-309

18 Loewenich, V. von, K. Riegel, K.H. Wulf: Transport der Risikogeborenen oder der Risikoschwangeren? gynäkol prax. 6 (1982) 233-238

19 McCormick, M.C., S. Shapiro B.H. Starfield: The regionalizatio of perinatal services. Summary of evaluation of a national demonstration program. J. Am. Med. Assoc. (JAMA) 253 (1985) 799-804

20 Modanlou, H.D., W. Dorchester, R.K. Frecman, C. Rommal: Perinatal transport to a regional perinatal zenter in a metropolitan area: Maternal versus neonatal trarisport. Am. J. Obstet. Gynecol. 138 (1980) 1157-1164

21 Obladen, M.; U. Heemann, K.H. Hennecke, L. Hanssler: Ursachen neonataler Letalität 1981-1983: eine regionale Analyse. Z. Geburtsh. U. Perinat. 189 (1985) $181-187$

22 Obladen, $M$. Das Risiko, geboren zu werden. Forum der Kinderheilk. 1 (1985) 17-22

23 Riegel, K., H.K. Selbmann: Perinatale kindliche Mortalität versus Morbidität aus der Sicht des Kinderarztes. Archs. Gynecol. 228 (1979) 88-95

24 Riegel, $K$.: Betreuung von kranken und gefährdeten Neugeborenen. Gemeinsame Stellungnahme der Destschen Gesellschaft für Gynäkologie und Geburtshilfe, der Deutschen Gesellschaft für Kinderheilkunde, der Deutschen Gesellscahft für Perinatale Medizin und der Deutsch-Osterreichischen Gesellschaft für Neonatologie und Pädiatrische Intensivmedizin. Kinderarzt 15 (1984) 1219

25 Riegel, K., H.K. Selbmann, K. Österlund: Perinatalrisiken und kindliche Mortalität und Morbidität. Arvo-Ylppö-Studie. BPTBericht 5/85. Gesellschaft für Strahlen- und Umweltforschung, München 1985

26 Rosenblatt, R.A., J. Reinken, Ph. Shoemack: Is obstetrics safe in small hospitals? Evidence from New Zealand's Regionalised Perinatal System. Lancet II (1985) 429-439

27 Usher, R.: Changing Mortality Rates With Perınatal Intensive Care and Regionalization. Seminars in Perinatology 1 (1977) $309-319$

Prof. Dr. med. Volker von Loewenich, Abteilung für Neonatologie, Zentrum der Kinderheilkunde der Johann Wolfgang Goethe-Universität Theodor-Stern-Kai 7, D-6000 Frankfurt/Main 70 